Projets

de paysage

\section{Projets de paysage}

Revue scientifique sur la conception et l'aménagement de l'espace

$18 \mid 2018$

Paysage et didactique

\title{
Des controverses en éducation au paysage : quels enjeux éducatifs, finalités didactiques et modalités pédagogiques?
}

Controversies in landscape training: Educational challenges, didactic objectives and pedagogical objectives

\section{Virginie Albe}

\section{(2) OpenEdition}

\section{Journals}

Édition électronique

URL : http://journals.openedition.org/paysage/1011

DOI : 10.4000/paysage.1011

ISSN : 1969-6124

Éditeur :

École nationale supérieure du paysage de Versailles-Marseille, Institut national des sciences appliquées Centre Val de Loire - École de la nature et du paysage, École nationale supérieure d'architecture et de paysage de Bordeaux, École nationale supérieure d'architecture et de paysage de Lille, Agrocampus Angers

\section{Référence électronique}

Virginie Albe, «Des controverses en éducation au paysage : quels enjeux éducatifs, finalités didactiques et modalités pédagogiques ? », Projets de paysage [En ligne], 18 | 2018, mis en ligne le 06 juillet 2018, consulté le 28 novembre 2019. URL : http://journals.openedition.org/paysage/1011 ; DOI : 10.4000/paysage.1011

Ce document a été généré automatiquement le 28 novembre 2019.

Projets de paysage 


\title{
Des controverses en éducation au paysage : quels enjeux éducatifs, finalités didactiques et modalités pédagogiques?
}

\author{
Controversies in landscape training: Educational challenges, didactic objectives \\ and pedagogical objectives
}

Virginie Albe

1 Les controverses sont considérées comme une entrée possible pour une éducation au paysage (Cefaï et Trom, 2001; Kirat et Torre, 2008). Il s'agit alors de considérer le paysage comme un objet politique (Sgard, Fortin et Peyrache-Gadeau, 2010), porteur d'intérêts différents selon les groupes sociaux concernés, mais aussi de valeurs et d'idéologies, ce qu'une analyse sociologique et épistémologique des controverses permet d'identifier. Éduquer au paysage par ou dans des controverses paysagères vise ainsi à contribuer à la formation d'un acteur politique apte à développer une pensée autonome et aussi des actions propres au paysage. Une telle éducation politique au paysage est destinée à tous les acteurs intéressés par/dans la controverse. Elle n'est ainsi pas réduite à un public scolaire. Mais comment les curricula d'éducation et de formation intègrent-ils une telle éducation politique au paysage? Quel(s) rôle(s) joue la controverse en situation d'éducation ou de formation? Autrement dit, quels sont les finalités éducatives d'une étude de controverse paysagère, les enjeux d'apprentissage qui y sont rattachés, les modalités pédagogiques mises en œuvre, les apprentissages réalisés?

Dans cet article, nous avons tenté de répondre à ces questions en interrogeant la littérature scientifique dans plusieurs disciplines, géographie et aménagement, sciences sociales et sciences de l'éducation. Dans un premier temps, nous avons constitué un corpus principal de 44 articles scientifiques publiés en langues française et anglaise ayant pour objet l'étude de controverses paysagères et environnementales. Puis, dans un second temps, nous avons focalisé la discussion sur l'intégration des 
études de controverses dans le curriculum. Articulant contenus, formes et finalités des enseignements, le curriculum agit comme un régulateur symbolique, mettant en correspondance savoir, pouvoir et formation de la conscience (Bernstien, 1996 et 2007, p. 73). Porter attention au curriculum c'est ainsi considérer l'ensemble des processus de production et de transformation des savoirs en vue de former des personnes. Opter pour une entrée par le curriculum consiste à dépasser deux réductionnismes en sciences de l'éducation. D'une part, dans les approches sociologiques en éducation, peu d'attention est portée aux savoirs (Lahire, 2012), soit considérés comme donnés, soit le seul produit de luttes de pouvoir entre groupes sociaux (Frandji et Vitale, 2008; Stavrou, 2017). D'autre part, les didactiques des disciplines ont largement favorisé une perspective « internaliste » des savoirs, et peu interrogé la constitution des disciplines elles-mêmes.

3 Afin de nourrir une telle discussion sur l'intégration curriculaire des études de controverses, nous avons constitué, de façon indépendante du corpus principal, un corpus secondaire de 36 articles scientifiques ayant pour objet l'étude de controverses socioscientifiques. Cette approche a permis de documenter le(s) statut(s) de la controverse dans les domaines éducatifs d'éducation à l'environnement et d'éducation au développement durable, qui sont les plus présents dans l'enseignement secondaire et primaire en France, mais aussi d'éducation au paysage plus implicitement abordée dans ce même contexte éducatif. Une telle démarche a permis d'interroger l'étude de controverses paysagères en regard d'une littérature internationale (Sadler, 2004 et 2009) déjà fournie sur les modalités d'études de controverses socioscientifiques.

\section{Méthodologie}

\section{Constitution des corpus}

4 Deux corpus de textes ont été constitués à partir du portail de recherche bibliographique du CNRS (BibCNRS). La même démarche de constitution a été adoptée pour chaque corpus. Elle repose au départ sur une recherche par mots-clés.

5 Pour le corpus principal, nous avons interrogé ce portail de recherche bibliographique avec, dans un premier temps, les mots-clés "controverse paysagère » et obtenu 4 documents dont un mémoire d'HDR (Sgard, 2011) et une thèse de doctorat (Robert, 2011) en géographie ainsi qu'un article issu de cette thèse. Nous avons alors dans un deuxième temps étendu cette recherche bibliographique en utilisant les mots-clés " paysage » et « controverse ». Cela nous a fourni 156 documents en langues française, anglaise, espagnole et portugaise, publiés entre 1754 et 2017 dont 55 articles dans des revues scientifiques, 11 ouvrages et 8 documents électroniques.

6 Pour notre analyse, et pour des raisons pratiques, les articles en portugais (12) et en espagnol (1) ont été éliminés ainsi que les rapports d'enquêtes, comptes rendus de séminaires ou de colloques, et thèses. Puis dans un troisième temps, la lecture des résumés a permis d'éliminer les articles portant sur une analyse de représentations ou une analyse juridique, et les articles dans lesquels le terme « controverse » est utilisé en sous-titre pour préciser l'objet de la recherche portant sur une thématique autre, par exemple la responsabilité sociale des entreprises, le patrimoine, ou les changements climatiques. Le corpus ainsi constitué comporte alors 44 articles publiés dans des revues scientifiques, en langue française et anglaise. 
7 Pour le corpus secondaire, nous avons également interrogé le portail de recherche bibliographique du CNRS avec, dans un premier temps, les mots-clés "controverse socioscientifique ». Cela nous a fourni 19 documents en langue française, articles de revues, un ouvrage et des recensions afférentes. Nous avons alors dans un deuxième temps élargi la recherche bibliographique avec les mots-clés en langue anglaise « socioscientific issues » et recueilli 1044 documents publiés entre 1986 et 2017. Nous avons alors réduit la recherche à la période des dix dernières années, et obtenu ainsi 815 documents. En réduisant encore ce corpus à la présence des mots-clés dans le titre, nous avons obtenu 340 documents publiés de 2007 à 2017. Nous avons finalement retenu de ces 340 documents, les seuls 17 qui ont été publiés dans les meilleures revues du champ «science education »: 9 dans le Journal of research in science teaching, 6 dans Science Education, 2 dans Cultural Studies of Science Education. Au final, ce corpus secondaire contient 36 documents en langues française et anglaise.

\section{Analyse des corpus de textes}

Les textes ont été traités de manière empirique, avec une grille d'analyse élaborée $a$ priori. Cette grille comporte 9 critères de façon à identifier dans les documents les thèmes ou objets de controverses, les contextes d'étude de ces controverses (discipline, éducation à l'environnement ou au développement durable, projet éducatif, recherche...), les rôles attribués à ces controverses (contexte, objet ou moyen d'apprentissage ?), ainsi que les champs disciplinaires et les visées des recherches, les enjeux éducatifs et apprentissages réalisés, les méthodologies mises en œuvre et les résultats obtenus (tableau 1). Afin de documenter les questions de notre problématique sur les finalités éducatives des controverses, nous avons structuré notre revue de littérature à partir des rôles attribués aux controverses dans les recherches.

Tableau 1. Grille d'analyse des corpus

Thèmes ou objets de controverses

Contextes d'étude des controverses : discipline, éducation à l'environnement ou au développement durable, projet éducatif, recherche...

Rôles attribués aux controverses : contexte, objet ou moyen d'apprentissage

Champs disciplinaires des recherches

Visées des recherches

Enjeux éducatifs

Apprentissages

Méthodologies

Résultats 


\section{Éducation dans/par des controverses paysagères ?}

9 Un bref classement des 44 textes du corpus principal a été effectué à partir des mots les plus fréquemment utilisés selon le traitement statistique du portail de recherche bibliographique BibCNRS. Cela indique qu'il s'agit de recherches en géographie et aménagement, en sciences de l'environnement, en sciences sociales et en particulier sociologie, avec une approche interdisciplinaire ou multidisciplinaire, et qui portent le plus souvent sur les politiques et actions publiques, la décision et la gouvernance, le développement, les mouvements politiques et sociaux et la contre-expertise, le territoire, la biodiversité et les changements climatiques.

Figure 2. Mots les plus fréquemment utilisés dans les articles du corpus

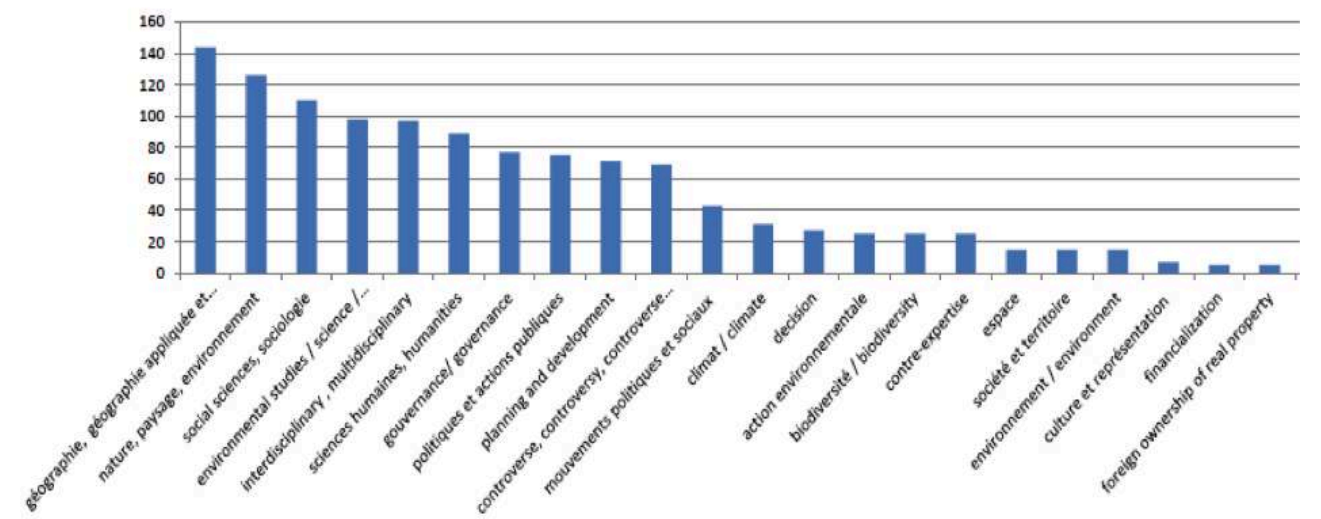

L'analyse des articles donne rapidement l'idée que la dimension éducative ou formatrice des controverses est peu abordée, en tant que telle, dans les recherches de notre corpus. Bien que non traitée de façon explicite dans les articles, cette dimension éducative est pourtant bien présente dans les controverses, notamment lorsque des projets d'installations techniques sont mis en débat par des non-spécialistes (ligne à haute tension dans November et al., 2004, infrastructures de transports terrestres dans Rocher, 2013; Jastremski, 2013 ; Brière, 2013 et ferroviaires dans Vandevelde, 2013, équipements énergétiques dans Lyrette et Simard, 2006). Il ressort de ces recherches que le discours des acteurs à propos de ces controverses ne peut être réduit à des jeux de pouvoirs qui se déploieraient sans mobilisation de savoirs. Au contraire, chaque protagoniste de la controverse développe des activités épistémiques, pour peser pleinement dans les débats et faire valoir ses arguments et intérêts. La nature des données en jeu et leurs mobilisations diverses dans les argumentations déployées dans la controverse sont aussi l'objet d'une appropriation par les acteurs. À ce titre, une activité didactique se développe dans ces controverses paysagères ou environnementales.

11 En outre, un potentiel éducatif est identifié lorsque des aménagements du milieu naturel engendrent une controverse paysagère (Auclair et Lasserre, 2013), et pourraient aussi alimenter des activités de médiation scientifique (Suchet, 2010). Or, dans le contexte éducatif français, le paysage est peu traité explicitement, l'accent étant plutôt mis sur les enjeux environnementaux ou l'éducation au développement durable (EDD), le paysage pouvant être implicitement pris en compte via des discussions sur le cadre de vie par exemple. 


\section{Des controverses socioscientifiques pour une éducation citoyenne}

12 L'analyse des 36 articles du corpus secondaire indique que les différentes recherches mobilisent la controverse de différentes façons, comme contexte, objet ou moyen d'apprentissage. Selon ces différents rôles attribués à la controverse, les pratiques, savoirs et compétences d'un citoyen et d'une citoyenne que l'étude de controverse vise à développer peuvent être identifiés à l'analyse.

\section{La controverse comme contexte d'apprentissage}

Il s'agit ici, dans les différentes recherches, de favoriser la compréhension des concepts mobilisés dans la controverse.

Par questionnaires, Fowler et Zeidler (2016) ont identifié les concepts scientifiques relatifs à la théorie de l'évolution mobilisés dans des controverses sociobiologiques par 52 étudiants de fin de premier cycle universitaire en biologie et dans d'autres disciplines. Ils ont en outre montré que l'acceptation de l'évolution comme théorie scientifique est un facteur déterminant dans la mobilisation de connaissances scientifiques dans les controverses sociobiologiques. Dans la controverse du changement climatique, Dawson (2015) a étudié la compréhension des éléments scientifiques d'élèves de lycée (grade 10, 14-15 ans) ${ }^{1}$. Si les élèves connaissent des concepts, ils méconnaissent parfois leurs relations et élaborent des conceptions alternatives mêlant effet de serre et couche d'ozone, différents types de gaz à effet de serre et types de radiations, climat et météo, pollution de l'air.

D'autres recherches portent sur l'impact d'activités didactiques spécifiquement élaborées pour favoriser la compréhension conceptuelle des élèves lors de l'étude de controverses. Ainsi, Jho, Yoon et Kim (2014) ont montré par questionnaires pré-postintervention auprès de 89 étudiants que leur compréhension de l'énergie nucléaire augmentait. Par ailleurs, la compréhension conceptuelle de la génétique par 92 élèves de lycée s'est aussi améliorée après une courte intervention de 3 séances de 50 minutes, chacune dans la recherche de Venville et Dawson (2010). En outre, cette amélioration est plus importante dans une classe ayant été explicitement formée à l'argumentation.

\section{La controverse comme objet d'apprentissage}

Dans les recherches de notre corpus qui considèrent la controverse comme objet d'apprentissage, les finalités éducatives consistent à développer chez les jeunes :

- une compréhension critique de la nature des expertises, des rôles et pouvoirs des experts, afin de produire leurs propres expertises dans des controverses telles que les changements climatiques, la dangerosité de la téléphonie cellulaire ou les nanotechnologies (Albe et Gombert, 2012 ; Pouliot, 2012) ;

- une complexification épistémologique, s'appuyant notamment sur une compréhension de la nature des expertises et de ce qui distingue les expertises des recherches, et amenant les jeunes à remettre en cause une vision strictement empirico-réaliste des sciences ; 
- une opinion informée sur ces questions, et une prise de décision documentée et raisonnée (Grace et al., 2015 ; Lundstrom et al., 2012).

\section{La controverse comme moyen pour l'éducation morale des jeunes}

17 Selon une telle perspective, il s'agit de mettre l'accent sur les dimensions morales et éthiques des controverses. Les recherches menées selon cette perspective d'éducation morale sont en partie conceptualisées à partir des stades du développement du raisonnement moral (Kohlberg, 1986), sur le modèle des stades de développement cognitif de Piaget. Mais alors que dans le paradigme Kohlbergien une prise de décision basée sur l'émotion représente un raisonnement moral sous-développé, dans ces recherches (Zeidler et al., 2009) aucun mode de prise de décision n'est disqualifié $a$ priori. Sont ainsi soulignés ici le rôle des émotions dans l'étude de controverses et l'importance de prendre en compte l'affect pour dépasser les aspects cognitifs dans l'éducation aux sciences.

\section{La controverse comme moyen de développer les compétences argumentatives des jeunes et/ou leur action sociale}

La controverse est aussi, dans nombre de recherches du corpus analysé, considérée comme un moyen pour faire débattre les élèves afin de développer leurs compétences citoyennes. Les dispositifs d'apprentissage mis en œuvre sont ici centrés sur la pratique du débat en contexte scolaire, dans un cadre adidactique de collecte de données aux seules fins de recherche, ou dans le cadre de projets éducatifs notamment interdisciplinaires, ou au sein de disciplines scientifiques. Les auteurs mettent alors l'accent sur la construction de situations scolaires en relation avec des situations sociales. Il peut s'agir de faire examiner aux élèves des problèmes authentiques, en résonance avec leur vie quotidienne et leur milieu et les engager dans l'élaboration d'une expertise, une prise de décision argumentée ou la participation à un débat entre experts.

19 Plus rarement, la pratique du débat avec les jeunes se déploie aussi dans l'espace public, lors par exemple de l'étude de l'aménagement d'un marais ou de la communication de résultats d'analyse de la qualité des eaux sur le territoire de l'école (Jimenez-Aleixandre et Pereiro-Munoz, 2002). La formation d'élèves-citoyens dépasse dans ce cas le cadre scolaire avec une participation sociale, ce qui rejoint les objectifs de la Convention européenne du paysage notamment. Il peut également s'agir de mettre en œuvre en classe des dispositifs d'apprentissage qui simulent des pratiques sociales, comme des débats publics ou des conférences de consensus. Ainsi ont été proposés des controverses pour l'action et pour le doute, la réflexion (que faire face à... ? quelle décision prendre pour... ?) avec l'intention de favoriser le développement de la pensée critique et la formation à l'action sociopolitique (Hodson, 2011). La participation ou la (re)configuration sociale nécessitent pour plusieurs auteurs de former les élèves à comprendre la nature des sciences et à évaluer les discours des experts sur des controverses socioscientifiques, alors aussi objets d'apprentissage, comme nous l'avons vu plus haut.

20

Dans les recherches, les élèves se sont montrés aptes à argumenter sur les controverses socioscientifiques soumises à leur étude (Evagorou et Osborne, 2013; Orlander et 
Lundegard, 2012), et à prendre des décisions (Grace et al., 2015 ; Lundstrom et al., 2012), soit en mobilisant un registre épistémologique dans lequel la preuve scientifique joue un rôle majeur, soit en se basant sur des considérations personnelles. Les considérations épistémologiques des élèves indiquent une représentation des sciences à saveur empirico-réaliste mais également des approches plus critiques des sciences, des pratiques d'expertise scientifique et des relations entre expertise et politique (Herman, 2015; Wu et Tsai, 2011). La mobilisation de connaissances scientifiques s'effectue en lien avec des considérations personnelles et les discours publics déployés dans la controverse (Rose et Calabrese Barton, 2012 sur la construction d'une usine électrique en ville).

\section{Discussion : intégration curriculaire des controverses?}

21 Notre analyse d'un corpus de recherches sur l'étude de controverses en éducation indique que la controverse est mobilisée selon les cas comme un contexte, un objet ou un moyen d'apprentissage. Lorsque la controverse est utilisée comme contexte à l'apprentissage de connaissances scientifiques, elle joue un rôle d'accroche motivante ou de prétexte à un apprentissage conceptuel. L'étude de controverses peut dans ce cas s'intégrer à un enseignement disciplinaire des sciences traditionnellement organisé en corpus de connaissances conceptuelles, et ceci sans que cela pose de nouveaux défis au contrat et à la coutume didactique, ni ne remette en cause plus largement la forme scolaire. La controverse est aussi un moyen de développer les compétences argumentatives des élèves dans nombre de recherches. Apparaît ici un risque de confiner la pratique du débat à un exercice scolaire, notamment si les dispositifs d'apprentissage mis en œuvre en classe sont peu intégrés au curriculum et/ou peu ancrés à des pratiques sociales, dont le débat démocratique. À l'inverse, la controverse est aussi un moyen pour viser une démocratisation des technosciences et de la démocratie (Roth et Désautels, 2002). Il s'agit ici de contribuer à une éducation pour l'action sociale et l'engagement. Selon une finalité émancipatoire, l'étude de controverses vise à donner pouvoir (empower) aux élèves. Ceux-ci sont d'ailleurs considérés comme de jeunes acteurs sociaux et politiques plutôt qu'envisagés selon leur seule dimension épistémique et de façon générique, pour participer aux débats publics et aux prises de décisions en matière technoscientifique ainsi qu'à la configuration de la société. La controverse (paysagère, environnementale, socioscientifique...) peut aussi être appréhendée selon cette même visée de reconfiguration sociale comme un objet d'apprentissage. Cela exige de "payer le prix" d'une exploration documentée, approfondie et outillée de la controverse, mobilisant à la fois du temps et les outils et concepts de l'analyse d'arguments, de documents et de médias, et de la sociologie des pratiques scientifiques.

22 Les controverses posent de nouvelles questions aux didactiques et plus largement aux recherches en éducation. Elles remettent en question le fonctionnement standard de la forme scolaire et formulent de nouvelles demandes exigeantes aux enseignants. Ainsi, par exemple, s'il paraît aisé d'intégrer des controverses à un enseignement disciplinaire en les utilisant comme une accroche motivante pour l'apprentissage de connaissances, il n'en est pas de même pour la perspective d'empowerment. Lorsque la controverse est objet d'étude, il s'agit bien d'ouvrir la « boîte noire » pour identifier les 
enjeux économiques, politiques, environnementaux, etc., les acteurs impliqués, leurs intérêts, leurs arguments et modes d'action. Une telle approche nécessite un outillage qui dépasse les concepts et méthodes d'une seule discipline, et l'intégration de controverses dans le curriculum constitue une problématique qui reste à approfondir. Parmi les questions qui se posent, on peut s'interroger à partir de l'expérience du courant des controverses socioscientifiques si une éducation aux/par des controverses ne risque pas une possible instrumentalisation par les disciplines pour tenter de relégitimer leur pertinence sociale par «arrimage » à des problèmes sociétaux. Nous nous demandons aussi quelles peuvent être les collaborations pluridisciplinaires, les mobilisations de savoirs disciplinaires (ces derniers pouvant être par exemple des ressources pour nourrir des débats sur des questions sociétales ou politiques), les modalités d'intégration formelle de controverses dans les «éducations à »... la citoyenneté, l'environnement, le développement durable?

À propos par exemple des rapports entre la géographie et l'éducation au développement durable, a par exemple été soulevée une instrumentalisation progressive des disciplines, "simples contributrices à un objectif qui les dépasse " (Vergnolle Mainar, 2011, p. 43). Pour Doussot (2015, p. 153) : «Les disciplines valent désormais moins pour leurs contenus à transmettre que comme des ressources au service de problèmes actuels. " La recherche empirique de Doussot (2015) a en outre montré une difficulté pour les élèves à synthétiser des éléments disciplinaires hétérogènes, activité habituellement menée par l'enseignant. Le traitement de questions de nature politique, pour l'éducation au développement durable, comme dans le cas de controverses visant un empowerment citoyen, implique en effet de faire intervenir divers types de savoirs : des savoirs disciplinaires, des expertises, des savoirs issus de l'expérience ou de mondes professionnels variés, des savoirs produits par des groupes sociaux non académiques, et donc non reconnus socialement comme producteurs de savoirs (Albe, 2009). Une telle diversité des types de savoirs réinterroge le débat dans les didactiques portant sur la nature des savoirs sur lesquels fonder les savoirs scolaires, transposition des savoirs des disciplines académiques ou références à des pratiques sociales.

\section{Conclusion}

Afin d'interroger les potentialités didactiques de controverses paysagères, nous avons analysé deux corpus d'articles scientifiques, sur les controverses paysagères pour le premier et sur des controverses socioscientifiques pour le second. Notre analyse indique que la dimension didactique de controverses paysagères, bien que présente ou potentielle dans les recherches du premier corpus, n'est pas explicitement travaillée. Des apprentissages se réalisent pourtant lorsque par exemple des non-spécialistes s'approprient les données mobilisées dans des controverses sur des projets d'infrastructures de transport ou d'énergie. Ces acteurs "profanes" font aussi l'apprentissage de divers usages de ces données dans les arguments déployés par les autres protagonistes et développent une activité épistémique spécifique pour participer aux débats. En outre, des recherches dans ce premier corpus identifient une dimension didactique potentielle à des controverses d'aménagements du milieu naturel via une médiation scientifique. 


\section{d'apprentissage selon un enjeu d'éducation politique constituent selon nous une} approche fructueuse pour viser une démocratisation des expertises, des politiques technoscientifiques et contribuer à l'action sociale et à l'engagement. Mais l'étude de ces controverses socioscientifiques dans le cadre de telles institutions d'éducation heurte le fonctionnement standard de la forme scolaire et pose de nouveaux défis de positionnement aux enseignants. À la fois spécialistes d'une discipline (voire de deux), profanes vis-à-vis de la controverse et animateurs des débats entre élèves, cela remet en cause la coutume didactique dans laquelle l'enseignant maîtrise les savoirs. L'intégration curriculaire de telles controverses soulève des questions sur les différents types de savoirs qui peuvent être scolarisés. La question de l'intégration curriculaire des controverses rejoint en outre des débats qui se déploient dans la littérature scientifique en éducation sur les modalités de collaborations pluridisciplinaires, les relations entre disciplines et éducation au développement durable, et entre savoirs et compétences.

28 À partir de ces analyses de la littérature, il nous apparaît que la controverse paysagère pourrait constituer une entrée prometteuse pour une éducation politique. Considérer le paysage tel un objet politique comme le proposent Sgard, Fortin et Peyrache-Gadeau (2010) nous semble permettre d'éviter certains risques que nous avons soulignés plus haut à partir de nos analyses de la littérature. Ainsi par exemple, dans la mesure où le paysage ne nous semble pas de nature à pouvoir être réduit à un objet strictement scolaire, il y a peu de risques à confiner la controverse à un moyen scolaire pour faire pratiquer le débat ou l'argumentation sans lien avec des pratiques sociales. Dans la même veine, appréhender le paysage comme un objet politique oriente vers une approche interdisciplinaire ou multidisciplinaire, et éloigne ainsi le risque de limiter la controverse à un contexte pour l'apprentissage de contenus disciplinaires.

$\mathrm{Au}$ contraire, des controverses paysagères pourraient constituer des objets d'apprentissage qui mobilisent une pluralité de savoirs, dont les savoirs scientifiques, techniques, économiques, politiques... comme dans le cas des controverses socioscientifiques. Mais d'autres types de savoirs pourraient aussi être convoqués dans l'étude de controverses paysagères, comme des savoirs issus de l'expérience ou de pratiques professionnelles, des considérations esthétiques, et des émotions. Ceci pouvant se faire, au contraire des controverses socioscientifiques, sans ouvrir un débat sur la légitimité de ce type de savoirs. En outre, des controverses paysagères pourraient 
servir de moyen pour l'action sociale dans la mesure où il serait débattu des politiques et des actions publiques, de la décision et de la gouvernance, du développement, de la nature des expertises, du territoire, comme l'indiquent les thèmes identifiés dans le premier corpus étudié.

\section{BIBLIOGRAPHIE}

Albe, V. et Gombert, M.-J., « Students' communication, argumentation and knowledge in a citizens' conference on global warming ", Cultural Studies in Science Education, vol. 7, $\mathrm{n}^{\circ}$ 3, 2012, p. 659-681.

Albe, V., Enseigner des controverses, Rennes, PUR, 2009.

Auclair, A. et Lasserre, F., « Aménagements, politiques et conflits sur l'eau en Afrique de l'Ouest ", Vertigo. La revue électronique en sciences de l'environnement, vol. $13, \mathrm{n}^{\circ} 2$, mis en ligne en octobre 2013, URL : http://vertigo.revues.org/13994.

Bernstein, B., Pédagogie, contrôle symbolique et identité. Théorie, recherche, critique, (1996), Laval, Presses Universitaires de Laval, 2007.

Brière, L. (2013). «L'exercice du jugement dans les débats publics expertisés : le cas de la reconstruction de l'échangeur Turcot à Montréal », Vertigo. La revue électronique en sciences de l'environnement, vol. 13, $\mathrm{n}^{\circ}$ 2, mis en ligne en octobre 2013, URL : http://vertigo.revues.org/14000.

Cefaï, D. et Trom, D., Les Formes de l'action collective. Mobilisation dans des arènes publiques, Paris, Éditions de l'École des hautes études en sciences sociales, 2001.

Dawson, V., « Western Australian High School Students' Understandings about the Socioscientific Issue of Climate Change », International Journal of Science Education, vol. 37, n 7, 2015, p. 1024-1043. Doussot, S., « Enjeux didactiques de la recomposition des disciplines scolaires en histoire et géographie ", dans Audigier, F., Sgard, A. et Tutiaux-Guillon, N. (dir.), Sciences de la nature et de la société dans une école en mutation, Bruxelles, De Boeck, 2015, p. 151-161.

Evagorou, M. et Osborne, J., « Exploring young students' collaborative argumentation within a socioscientific Issues ", Journal of Research in Science Teaching, vol. 50, nº 2, 2013, p. 209-237.

Fowler, S. R. et Zeidler, D. L., « Lack of Evolution Acceptance Inhibits Students' Negotiation of Biology-based Socioscientific Issues », Journal of Biological Education, vol. 50, n 4, 2016, p. 407-424. Frandji, D. et Vitale, P. (dir.), Actualité de Basil Bernstein. Savoir, pédagogie et société, Rennes, PUR, 2008.

Grace, M., Lee, Y. C., Asshoff, R., Wallin, A., « Student Decision-Making about a Globally Familiar Socioscientific Issue: The Value of Sharing and Comparing Views with International Counterparts ", International Journal of Science Education, vol. 37, n 11, 2015, p. 1855-1874.

Herman, B. C., « The Influence of Global Warming Science Views and Sociocultural Factors on Willingness to Mitigate Global Warming ", Science Education, vol. 99, n 1, 2015, p. 1-38. 
Hodson D., Looking to the future: Building a curriculum for social activism, Rotterdam, Sense Publishers, 2011.

Jastremski, K., « Controverses entourant les projets d'infrastructures de transport dans la région de Montréal : quel rôle pour les experts? ", Vertigo. La revue électronique en sciences de l'environnement, vol. 13, $\mathrm{n}^{\circ}$ 2, mis en ligne en octobre 2013, URL : http://vertigo.revues.org/14178.

Jho, H. Yoon, H-G. et Kim, M., « The Relationship of Science Knowledge, Attitude and Decision Making on Socio-Scientific Issues: The Case Study of Students' Debates on a Nuclear Power Plant in Korea », Science \& Education, vol. 23, n 5, 2014, p. 1131-1151.

Jimenez-Aleixandre, M.-P. et Pereiro-Munoz, C., « Knowledge Producers or Knowledge Consumers? Argumentation and Decision Making about Environmental Management », International Journal of Science Education, vol. 24, $\mathrm{n}^{\circ} 11,2002$, p. 1171-1190.

Kirat, T. et Torre, A., Territoires de conflits. Analyse des mutations de l'occupation de l'espace, Paris, L'Harmattan, 2008, 324 p.

Kohlberg, L., "A current statement on some theoretical issues ", dans Modgil, S. et Modgil, C. (eds.), Lawrence Kohlberg: Consensus and controversy, Philadelphia, The Falmer Press, 1986, p. $485-546$.

Lahire, B., « De la division du travail scientifique : les rapports entre la didactique et la sociologie en période d'hyperspécialisation ", dans Losego, P. (dir), Actes du colloque sociologie et didactiques: vers une transgression des frontières?, Lausanne, HEP du Canton de Vaud, 2012, p. 48-59.

Lundstrom, M., Ekborg, M. et Ideland, M., « To Vaccinate or Not to Vaccinate: How Teenagers Justified Their Decision », Cultural Studies of Science Education, vol. 7, n 1, 2012, p. 193-221.

Lyrette, E. et Simard, J.-G., « L'action collective et la perception des acteurs face à la mise en place d'équipements énergétiques : les cas de Gentilly-2 et de la centrale de cogénération de Bécancour ", VertigO. La revue électronique en sciences de l'environnement, vol. 7, n 1 , mis en ligne en avril 2006, URL : http://vertigo.revues.org/2064.

November, V., D’Alessandro-Scarpari, C. et Remy, E., « Un lieu en controverse : une controverse qui fait lieu(x)», Norois, vol. 193, n 4, 2004, p. 91-102.

Orlander, A. et Lundegard, I., « « It's Her Body ». When Students' Argumentation Shows Displacement of Content in a Science Classroom ", Research in Science Education, vol. 42, $n^{\circ}$ 6, 2012, p. 1121-1145.

Pouliot, C., « Et pourquoi pas une éducation aux sciences qui aborde la participation des acteurs sociaux aux controverses sociotechniques?", Revue canadienne d'enseignement des sciences, des mathématiques et des technologies, vol. 12, n 4, 2012, p. 367-379.

Robert, A., « Dynamiques paysagères et guerre dans la province de ThừaThiên-Huế (Viêt Nam central), 1954-2007 : entre défoliation, déforestation et reconquêtes végétales ", thèse de doctorat de l'université Paris 4, 2011.

Rocher, L., « Infrastructure urbaine, planification et controverse : le projet différé d'un contournement routier au sud d'Angers ", Norois, vol. 227, n 2, 2013, p. 83-96.

Rose, S. R. et Calabrese Barton, A., « Should Great Lakes City build a new power plant? How youth navigate socioscientific issues ", Journal of Research in Science Teaching, vol. 49, n 5, 2012, p. 541-567.

Roth, W. M. et Désautels, J. (eds.), Science Education as/for Social Action, New York, Peter Lang, 2002. 
Rudaz, G. et Sgard, A. (dir.), « Les dimensions politiques du paysage », Géo-Regards, n 8, Neuchâtel, 2016.

Sadler, T. D., « Situated learning in science education: Socio-scientific issues as contexts for practice », Studies in Science Education, vol. 45, n 1, 2009, p. 1-42.

Sadler, T. D., « Informal reasoning regarding socioscientific issues: a critical review of research », Journal of Research in Science Teaching, vol. 41, n 5, 2004, p. 513-536.

Sgard, A., « Le partage du paysage. Géographie », université de Grenoble, 2011.

Sgard, A., Fortin, M.-J. et Peyrache-Gadeau, V., « Le paysage en politique », Développement durable \& territoires, vol. 1, $\mathrm{n}^{\circ}$ 2, mis en ligne en 2010, p. 1-11, URL : http://journals.openedition.org/ developpementdurable/8522.

Stavrou, S., L'Université au diapason du marché. Une sociologie du changement curriculaire dans les universités françaises, Louvain-La-Neuve, Academia, 2017.

Suchet, A., «Le site touristique aménagé des gorges de la Fou en Pyrénées françaises », Téoros, vol. $29, \mathrm{n}^{\circ} 2,2010$, p. $44-54$.

Vandevelde, J.-C., «L'outarde et le TGV : une controverse sur les compensations pour atteintes à la biodiversité », Vertigo. La revue électronique en sciences de l'environnement, vol. 13, $\mathrm{n}^{\circ} 2$, mis en ligne en octobre 2013, URL : http://vertigo.revues.org/14040.

Venville, G. J. et Dawson, V., « The Impact of a Classroom Intervention on Grade 10 Students' Argumentation Skills, Informal Reasoning, and Conceptual Understanding of Science ", Journal of Research in Science Teaching, vol. 47, $\mathrm{n}^{\circ}$ 8, 2010, p. 952-977.

Vergnolle Mainar, C., La Géographie dans l'enseignement, une discipline en dialogue, Rennes, PUR, 2011.

Wu, Y.-T. et Tsai, C.-C., « High School Students' Informal Reasoning Regarding a Socio-Scientific Issue, with Relation to Scientific Epistemological Beliefs and Cognitive Structures ", International Journal of Science Education, vol. 33, n 3, 2011, p. 371-400.

Zeidler, D. L., Sadler, T. D., Applebaum, S. et Callahan, B. E., « Advancing reflective judgment through socioscientific issues ", Journal of Research in Science Teaching, vol. 6, n 1, 2009, p. 74-101.

\section{NOTES}

1. 438 questionnaires et 20 entretiens.

\section{RÉSUMÉS}

Dans ce texte, nous avons tenté de mieux cerner les différents rôles attribués aux controverses lorsque celles-ci sont mobilisées à des fins d'éducation au paysage-environnement et aux sciences en vue de développer la citoyenneté. Nous avons réalisé pour cela deux revues de littérature en géographie et aménagement, sciences sociales et sciences de l'éducation. À partir du portail de recherche bibliographique du CNRS, deux corpus différents ont été constitués. Un corpus 
principal ayant pour objet l'étude de controverses paysagères et environnementales comporte 44 articles scientifiques publiés en langues française et anglaise. Un corpus secondaire ayant pour objet l'étude de controverses socioscientifiques contient 36 articles scientifiques publiés en langues française et anglaise. Nous avons discuté les modalités d'intégration curriculaire de controverses selon les différents rôles qui leur sont attribués dans les recherches, contexte, objet ou moyen d'apprentissage.

In this article, we have attempted to more clearly define the different roles assigned to controversies when these are used in teaching landscape architecture and science in order to develop a sense of citizenship. To do so we conducted two literature reviews in geography and urban planning and in social sciences and educational sciences. By using the CNRS bibliographical portal two different corpuses were constituted. A main corpus intended for the study of landscape controversies, comprising 44 scientific articles published in French and English, and a secondary corpus for the study of socio-scientific controversies, comprising 36 articles published in French and English. We discussed the modalities for the integration within the curriculum of controversies according to the different roles assigned to them in terms of educational research, contexts, objectives and means.

INDEX

Mots-clés : paysage, controverse, curriculum, didactique, éducation à l'environnement, EDD

Keywords : landscape, controversy, curriculum, didactics, environmental education, EDD

\section{AUTEUR}

\section{VIRGINIE ALBE}

Virginie Albe est professeure en éducation aux sciences et aux techniques à l'École normale supérieure de Cachan.

virginie.albe[at]ens-paris-saclay[dot]fr 